\title{
THE DESIGN OF AN AUTONOMIC CONTROLLER FOR SELF-MANAGED EMERGENCY DEPARTMENTS
}

\author{
Serene Almomen ${ }^{1}$ and Daniel A. Menascé ${ }^{2}$ \\ ${ }^{1}$ Volgenau School of Engineering, George Mason University, 4400 University Drive, Fairfax,VA 22030, U.S.A. \\ ${ }^{2}$ Department of Computer Science, George Mason University, 4400 University Drive, Fairfax, VA 22030, U.S.A. \\ \{salmome1, menasce\}@gmu.edu
}

\begin{abstract}
Keywords: Autonomic Computing, Healthcare, Emergency Department, Quality of Service, Length of Stay, Selfmanaged system, Simulation, Combinatorial Search

Abstract: Improving the operational efficiency of an Emergency Department (ED) is a complex task due the very large number of ED configurations that involve human and physical resources and due to the unpredictable nature of the ED's workload. Thus, managing the performance of EDs becomes difficult and expensive when carried out by human beings alone. A new approach, called self-managed EDs, consists of building into the ED the mechanisms required to self-adjust the ED's configuration parameters so that its Quality of Service (QoS) is constantly met. This paper presents the design of an autonomic controller for a self-managed Emergency Department and evaluates its effectiveness in maximizing a utility function of the ED subject to cost-constraints. The controller uses a combination of combinatorial search techniques with simulation models. Experimental results illustrate the operation of the controller and how it reacts to variations of patient interarrival times.
\end{abstract}

\section{INTRODUCTION}

In hospitals all over the country, healthcare Emergency Departments are known to be dynamic in nature making them complex environments to manage. The complexity stems from the sudden changes to the workload of an ED due to emergencies such as fire, natural disasters, and terrorist attacks that are hard to predict. An ED consists of both human (e.g., doctors, nurses, and technicians) and physical (e.g., X-ray machines and CT-scan) resources and is one of the most critical units in any healthcare organization. Therfore, it is vital to improve its efficiency to increase patient satisfaction and reduce operational cost.

Quality improvement principles and benchmarking for EDs are increasingly becoming important for the success of hospitals. Emergency physicians and nurses are now being asked to provide safe, timely, efficient, and cost-effective care. Many QoS metrics are currently being collected at EDs to proactively assess performance and identify opportunities for quality improvement. These QoS metrics, also known as Core Measures, can be time measures or proportion measures (Welch et al., 2006). Time measures include arrival time to first seen by a doctor, disposition to discharge, and overall patient length of stay (LOS). Proportion measures include things like num- ber of patients who left before they were supposed to, hospital diversion, and ED patient flow (ACEP, 2010).

It is widely known that EDs suffer from crowding problems that impact the performance and QoS provided to patients. Many studies have been conducted to find the cause of crowding in EDs and some solutions have been suggested. For example, calculus and chaos theory have been used to model ED crowding and to identify variables and formulas that can predict when orderly patient flow succumbs to crowding (Fields, 2003). In a related attempt, Asplin et. al. (Asplin et al., 2003) present a conceptual model that partitions ED crowding into three interdependent components (input, throughput, and output) which exist within an acute care system that delivers unscheduled care. The goal of this model is to provide a practical framework to faciliate ED crowding solutions. Another study used both log-rank tests and time-towait (survival) proportional-hazard regression models to determine variables affecting ED QoS metrics such as door-to-doctor and dwell times (Arkun et al., 2009). Results showed that factors such as triage category, ED occupancy, and day of the week contribute to decreased patient flow through the ED. Roberts et. al. suggest the use of simulation modeling combined with Six Sigma techniques to address issues of length of stay (LOS) and patient satisfaction in the 
ED (Roberts et al., 2006).

Managing the performance of complex environments, such as an ED, is difficult and expensive when carried out by human beings alone. A new approach, called self-managed ED, is discussed here. The paper discusses the mechanisms required to self-adjust the configuration parameters of an ED so that its QoS goals are constantly met. In (Almomen and Menascé, 2011) we discussed the potential benefits of applying autonomic computing (Huescher and McCann, 2008) techniques to design self-managed EDs. In this paper we provide a detailed design of an autonomic controller for a self-managed ED. Our approach combines an ED simulator with combinatorial search techniques (Rayward-Smith et al., 1996) to design controllers that run periodically (e.g., every few hours) to determine the best possible configuration for an ED given its current and predicted workload. The paper also demonstrates the operation of the autonomic controller as it maximizes a utility function of the ED subject to cost-constraints.

The rest of the paper is organized as follows. Section 2 presents the architecture and the algorithm used by the ED controller of the self-managed ED. Section 3 presents the experimental setting and the next section describes the results under various circumstances to illustrate the operation of the method. Finally, Section 5 presents some concluding remarks.

\section{CONTROLLER APPROACH}

Our framework, discussed initially in (Almomen and Menascé, 2011), consists in developing an autonomic controller for a self-managed ED system that can regulate and maintain itself with minimal human intervention. This is ideal in an ED environment since the goal is to create a system that is able to adapt to a constantly changing environment (such as patient flow, workload, and resource availability) in a way that preserves given operational goals (such as performance goals or QoS goals).

\subsection{The Control Loop}

This framework, implements the MAPE-K (Monitor/ Analyze/ Plan/ Execute - Knowledge) model (Kephart and Chess, 2003) in an ED environment as shown in Fig. 1. Within the ED context, autonomic managers define a control loop (the MAPE-K loop) that continuously monitor the environment and handles events that need action to be taken. Changes are made through action operations. Sensors determine the state of the managed ED resources and action operations may change the current state. The entire ED environment is a set of managed resources (e.g., doctors, nurses, lab technicians, X-ray machines, CTscan machines, beds). Autonomic managers continuously monitor the system and record the values of various performance metrics (e.g., Length of Stay, throughput, and utilization of various resources). A group of stakeholders (e.g., managers and executives) define a utility function to be maximized. This function measures how well the ED is meeting its goals and is a function of its various performance metrics. For example, the utility of the ED decreases as the Length of Stay (LOS) increases and increases as its throughput increases. Based on the defined utility function, the autonomic manager then plans and executes any specific actions needed to maximize the utility function and optimize pertinent QoS metrics. The steps of monitoring, analyzing, planning, and executing may be executed concurrently.

It is worth noting that our model takes into account the Human-in-the-loop (HITL) element (Parasuraman et al., 2000) (see Fig. 1). This means that in order for the ED autonomic manager to work sucessufully, human interaction is required. The resource in charge of managing the ED, such as a charge nurse, will be part of the decision making process of the autonomic manager and will be able to change the outcome, or reconfiguration commands, of the autonomic manager. HITL is important in our model because we realize that in the ED, an expert nurse can influence the outcome of the system in a way that is difficult if not impossible to autonomically reproduce exactly. HITL in the ED environment also readily allows for the identification of problems and requirements that may not easily be identified by other means of the system. Utilizing HITL provides a more realistic implementation approach to a self-managed ED.

\subsection{Architecture of the Controller}

Figure 2 shows details of the autonomic manager in an ED. The ED autonomic manager is based on the notion that the ED is enhanced with an ED controller that monitors the ED performance, monitors the resource utilization of the various resources of the ED, and executes, at regular intervals called controller intervals $(\mathrm{CI})$, a controller algorithm to determine the best configuration for the ED. As a result of running the controller algorithm, reconfiguration options are generated that will help charge nurses change the ED configuration to maintain optimal QoS. As shown in Fig. 2, the ED controller has four main components: Utility Function Computation, ED Simulator, Workload Analyzer, and Controller Driver. 


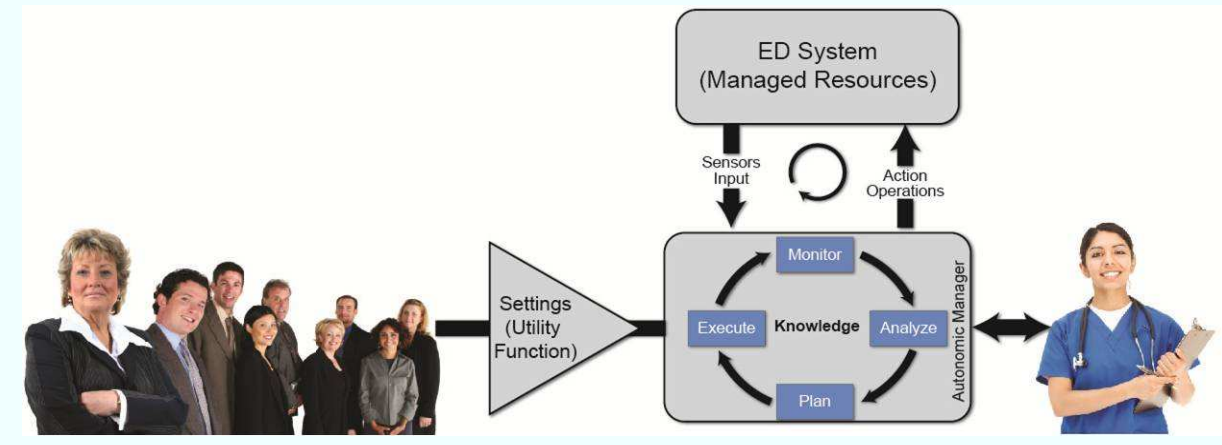

Figure 1: ED Autonomic Control Loop.

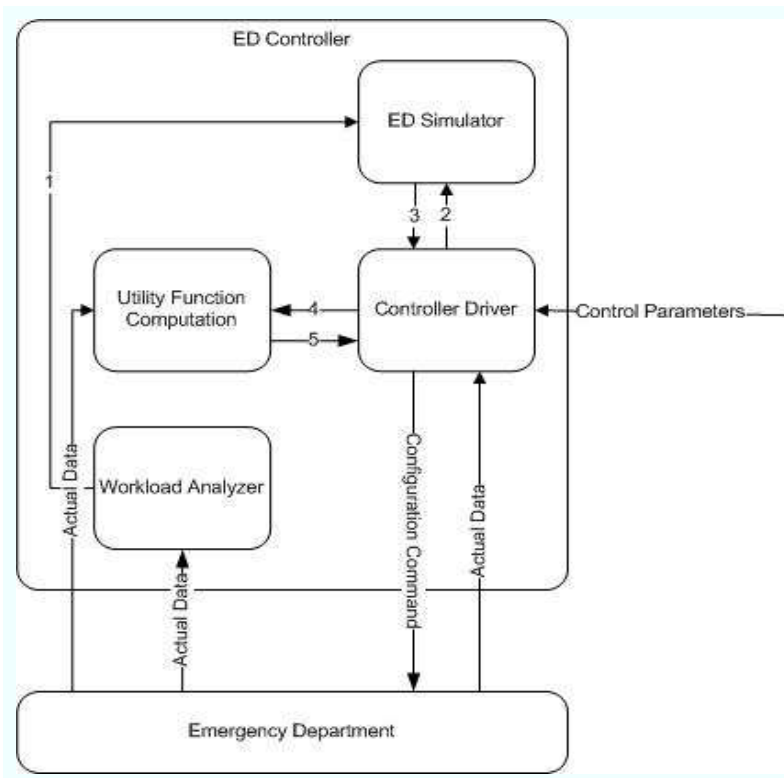

Figure 2: Architecture of the Autonomic Controller for the ED.

The Utility Function Computation component collects QoS metrics from the ED, such as resource utilization and patient length of stay (LOS), to compute the ED utility functions. The utility function is the goal to be optimized by the controller. The Workload Analyzer component analyzes the stream of arriving patients and forecasts the workload intensity values (e.g., average arrival rates) for the forthcoming periods. The predicted workload intensity values (1) computed by this component are used as input parameters to the ED Simulator. The ED Simulator receives requests (2) from the Controller Driver to run the ED Simulator corresponding for a specific configuration of the ED. The ED Simulator takes as input parameters configuration parameter values (2), and worload intensity values (1). The output of the ED Simulator is a resulting set of QoS values (3) for the configuration used as input by the Controller Driver. The Controller Driver then sends a request (4) to the Utility Function Computation component to compute the value of the utility function for the QoS values obtained from the ED Simulator. The corresponding value of the utility function (5) is returned to the Controller Driver. This last two steps are repeated for every point in each neighborhood visited by the Controller Driver, which executes a combinatorial search algorithm to determine the optimal configuration for the ED.

At the begining of each controller interval, the Controller Driver runs the combinatorial search engine, which takes into account the desired utility function and performs a combinatorial search (e.g., beam search or hill-climbing) (Rayward-Smith et al., 1996) of the state space of possible configuration points in order to find a near-optimal configuration. The QoS values have to be computed by the ED simulator for each point in the space of configuration points examined by the Controller Driver. Once the Controller Driver determines the best configuration for the workload intensity levels provided by the Workload Analyzer, it sends reconfiguration commands to the ED.

The Control Parameters shown in Fig. 2 provide for Controller Driver's settings such as the maximum number of configuration points to be searched before the search engine stops and the length of the controller interval (CI).

\subsection{Combinatorial Search Engine}

This section describes the combinatorial search algorithm used by the ED Controller. This algorithm, which is based on hill-climbing (RaywardSmith et al., 1996), is used to obtain a near-optimal configuration $\vec{C}=\left(c_{1}, \ldots, c_{p}\right)$ for the ED without performing an exhaustive search of the space of all pos- 
sible configurations.

We consider that the patients that arrive at the ED are categorized into tracks according to the resources they need and their workflow through the ED.

Let $\vec{W}$ be the vector of workload intensity (e.g., patient average arrival rate) values for each track. Let $\vec{C}_{\text {curr }}$ be the vector of current configuration parameters for the ED and let $\vec{C}_{\text {fin }}$ be the vector of final configuration parameters for the ED determined by the combinatorial search algorithm. In the example described here, a configuration vector $\vec{C}$ is a tuple $(n, d, t)$ where $n$ is the number of nurses, $d$ the number of doctors, and $t$ the number of lab technicians.

Let $U(\vec{Q})=f(\overrightarrow{\mathcal{W}}, \vec{C})$ be the value of the utility of the ED as a function of a vector $\vec{Q}$ of QoS values for the ED. The values in $\vec{Q}$ for a given configuration $\vec{C}$ and workload intensity vector $\overrightarrow{\mathcal{W}}$ are computed by a function $f$, which implements a simulation of the ED. In the example presented in this paper, we use LOS as the single QoS metric. Thus, $\vec{Q}=(L O S)$.

The utility function, $U(L O S)$, used in this paper follows a sigmoid curve of the type

$$
U(L O S)=K \frac{e^{\alpha(\beta-L O S)}}{1+e^{\alpha(\beta-L O S)}}
$$

where $K$ is a normalizing factor equal to

$$
K=\left(1+e^{\alpha \beta}\right) / e^{\alpha \beta}
$$

and $\beta$ is the QoS goal for the LOS, which is equal to 90 minutes in our experiments, and $\alpha$ is a sensitivity parameter that defines the sharpness of the curve. Sigmoids are commonly used as utility functions when the QoS metric is LOS or response time. We used $\alpha=0.05$ in our experiments. Note that the utility is a dimensionless quantity in the interval $[0,1]$.

Figure 3 depicts $U(L O S)$ according to Eq. (1) and the parameters defined above. As it can be seen, the utility is 1 when the LOS is zero. As the LOS increases, the utility decreases asymptotically to zero. When the LOS is equal to its QoS goal, the utility is equal to 0.5 . Note that any other utility function defined by the stakeholders could be used.

Each configuration $\vec{C}$ has a cost $\mathcal{C}(\vec{C})$ associated to it. The optimization problem implemented by the combinatorial search engine can be formally defined as

$$
\begin{array}{ll} 
& \text { Maximize } U(\operatorname{LOS})=f(\overrightarrow{\mathcal{W}}, \vec{C}) \\
\text { s.t. } & c(\vec{C}) \leq C O S T_{\max } \\
& c_{1}^{\min } \leq c_{1} \leq c_{1}^{\max } \\
& \cdots \\
& c_{p}^{\min } \leq c_{p} \leq c_{p}^{\max }
\end{array}
$$

Hill-climbing works by analyzing the neighboring configurations of a configuration being visited, determining the one with the highest utility, and moving to that point as the next point to visit. Let $\mathcal{N}(\vec{C})$ be the set of neighbors of configuration $\vec{C}$ in the space of possible configurations for the ED.

Typically, a neighbor of a given configuration is defined by a small perturbation in one of the elements of that configuration (e.g., increasing or decreasing by one the number of nurses of a given type). In our example, the neighbors of a configuration $(n, d, t)$ are $(n \oplus 1, d, t),(n \ominus 1, d, t),(n, d \oplus 1, t),(n, d \ominus 1, t)$, $(n, d, t \oplus 1)$, and $(n, d, t \ominus 1)$. The operator $\oplus$ is defined as $x \oplus 1 \leftarrow x+1$ if $x<x_{\max }$ else $x \leftarrow x_{\text {min }}$. In other words, the addition wraps around to $x_{\min }$. Similarly, $\ominus$ is defined as $x \ominus 1 \leftarrow x-1$ if $x>x_{\min }$ else $x \leftarrow x_{\max }$.

The search engine algorithm is given below.

- Step 1: (Initialization): NumIterations $\leftarrow 0 ; \vec{C} \leftarrow$ $\vec{C}_{\text {curr }} ; U \leftarrow f(\overrightarrow{\mathcal{W}}, \vec{C})$.

- Step 2: Find the configuration $\vec{C}_{\max }$ that has the largest utility among the configurations in $\{\vec{C} \in$ $\left.\mathcal{N}(\vec{C}) \mid \mathcal{C}(\vec{C}) \leq C O S T_{\max }\right\}$.

- Step 3: $U_{\max } \leftarrow f\left(\overrightarrow{\mathcal{W}}, \vec{C}_{\max }\right)$

- Step 4: If $U_{\max }>U$ then

$$
\begin{aligned}
& \left\{\vec{C} \leftarrow \vec{C}_{\max } ;\right. \\
& \left.U \leftarrow U_{\max }\right\} \\
& \text { Else }\left\{\vec{C}_{\text {fin }} \leftarrow \vec{C} ; \text { Stop }\right\} ;
\end{aligned}
$$

- Step 5: NumIterations $\leftarrow$ NumIterations +1 ;

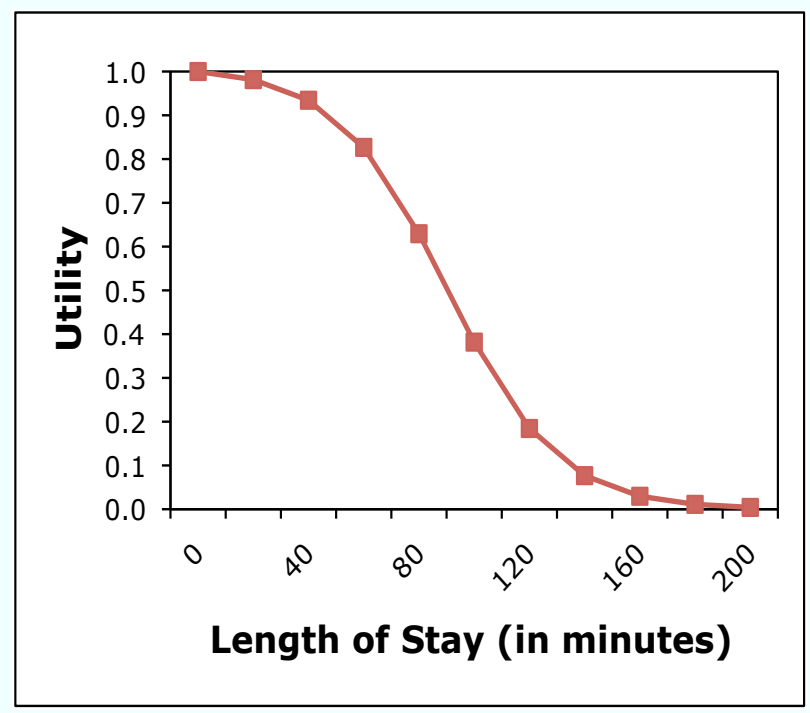

Figure 3: Utility as a function of Length of Stay (in minutes). 
- Step 6: If NumIterations $<$ MaxIterations then go to Step 2 else $\vec{C}_{\text {fin }} \leftarrow \vec{C}$.

The function $f$, i.e., the ED simulation, has to be run as many times as the number of points visited in the search space. This number is roughly equal to MaxIterations $\times \bar{N}$, where $\bar{N}$ is the average size of a neighborhood of a point in the configuration space. Assuming that each of the $p$ parameters of a point in the configuration space can vary in two possible ways, $\bar{N}$ can be estimated as $2 \times p$. One can reduce the number of times the simulation has to be run by storing the results of simulation runs in a hash table indexed by the configuration. This avoids re-running the simulation when a point is visited more than once, which occurs when neighborhoods overlap.

\section{THE EXPERIMENTAL SETTING}

In order to illustrate the operation of the controller described above, we developed an ED simulation model combined with a combinatorial search technique. Our experiment uses Flexsim HC Simulation Software (see www.flexsim. com/products/healthcare/) to simulate an Emergency Department (ED) that consists of a finite number of resources including beds, nurses, doctors, lab technicians, imaging machines, and labs (see Fig. 4). No patient is rejected from being seen in the ED. Arriving patients are required to register at check in.

A triage nurse performs a preliminary assessment of each ED patient. If no nurse is available, the patient waits in the waiting area. After triaging, an available staff nurse escorts the patient to the treatment area of the ED. If the treatment area is full, the patient waits in the triage area until the next room is available. In the treatment area, the patient is seen by a physician and a staff nurse for diagnosis. The ED patient flow is depicted in Fig. 5. Our experiment considers three patient tracks. A track is an easy way to break patients into different types that define possible pathways in the course of an ED visit. The different tracks are identified by the type of tests and/or screening, if any, the patient will need to take.

More specifically, the tracks considered here are:

- Basic: Patients in this track are discharged after being treated without the need for X-Ray screening or lab tests.

- X-Ray: Patients in this track need X-Ray screening before being discharged.

- X-Ray and Lab Test: Patients in this track need
Table 1: Initial Resource Settings.

\begin{tabular}{|c|c|}
\hline \hline Resource & Value \\
\hline \hline Nurse group & 4 \\
\hline Physician group & 3 \\
\hline Lab Technician group & 5 \\
\hline X-ray Technician group & 1 \\
\hline Bed & 4 \\
\hline X-ray machine & 1 \\
\hline Labs & 3 \\
\hline \hline
\end{tabular}

Table 2: Workload characteristics for the three tracks for experiments 1 and 2 .

\begin{tabular}{|l|c|c|}
\hline \hline Track & $\begin{array}{c}\text { Routing } \\
\text { Probability }\end{array}$ & $\begin{array}{c}\text { Interarrival } \\
\text { Time (min) }\end{array}$ \\
\hline \hline \multicolumn{3}{|c|}{ Experiment 1 } \\
\hline \hline Basic & $60 \%$ & 40 \\
\hline X-ray Experiment 2 \\
\hline X-Ray \& Lab Test & $20 \%$ & 45 \\
\hline \hline \multicolumn{2}{|c|}{ E } & 50 \\
\hline \hline Basic & $60 \%$ & 30 \\
\hline X-ray & $20 \%$ & 35 \\
\hline X-Ray \& Lab Test & $20 \%$ & 40 \\
\hline \hline
\end{tabular}

both X-Ray screening and three lab tests before being discharged.

The length of stay (LOS) for each patient can therefore be broken down into the waiting time at the waiting area, triage station, treatment area, and X-Ray or Lab (if required). Again, the LOS is the QoS metric measured in our experiment and used to calculate the utility function $U(L O S)$ to determine the optimal configuration of the ED. Additionally, we run the simulation with two different interarrival times (low and high intensity) and compare the results of the optimal ED configuration to illustrate our approach under varying interarrival times. The intearrival times and patient routing probabilities for the different tracks in each experiment are shown in Table 2.

In our experiment, we also assume that the number of X-ray technicians, beds, X-ray machines and labs is constant. Thus, the three configurable parameters to be changed by the contorller are the values of $n, d$, and $t$. The configurable parameters $(n, d, t)$ of the ED simulator are consequently adjusted dynamically at the end of every controller interval so that the utility is maximized. In addition, $\operatorname{COST} T_{\max }$ is set as $\$ 6,000.00 / \mathrm{hr}$ based on the following industry average fully loaded hourly rates: $\$ 75.00 / \mathrm{hr}$ for nurses, $\$ 250.00 / \mathrm{hr}$ for ED doctors, $\$ 30.00 / \mathrm{hr}$ for lab technician. In Flexsim Simulation, the Staffing Cost is calculated as: 


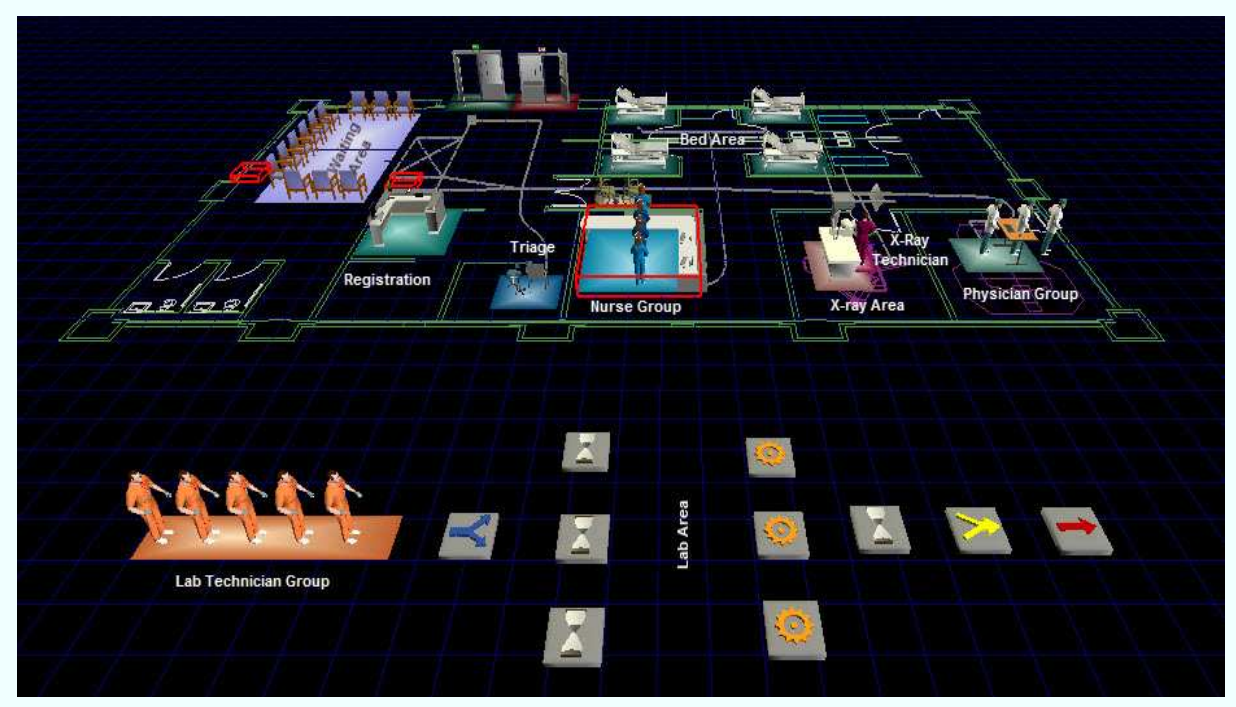

Figure 4: Simulated Emergency Department.

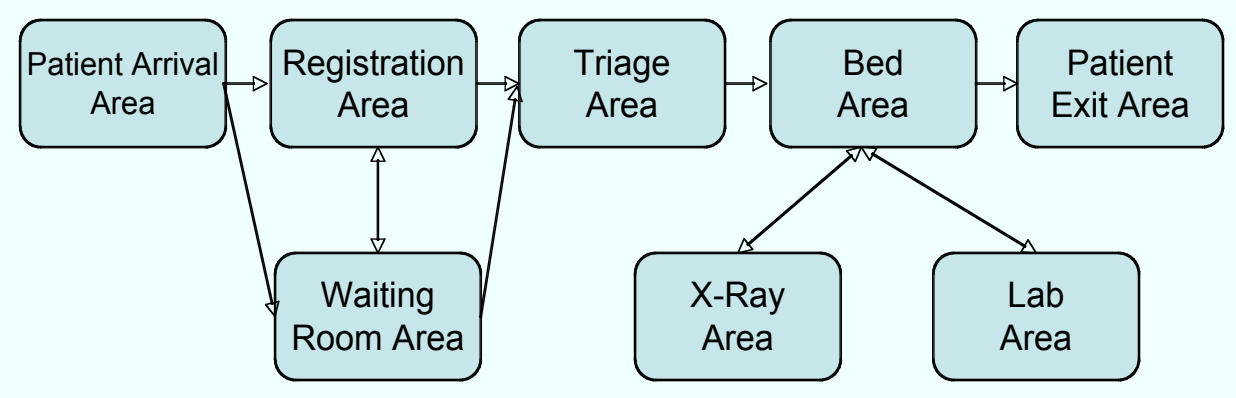

Figure 5: ED Model - Patient Workflow.

$$
\sum_{\forall \text { staff }} \text { StaffPayRate } \quad \times(\text { StaffTravelTime }+
$$

StaffServiceTime). (3)

According to Eq. (3), the hourly cost of the ED is a function of the time spent by its staff serving patients and therefore a function of the patient volume (Bamezai et al., 2005).

The initial values of the resources are shown in Table 1. The configurable parameters of the tuple $(n, d, t)$ are also limited by the following [min, $\max ]$ values:

- Nurse: $n \in[2,4]$

- Physician $d \in[1,3]$

- Technician $t \in[3,5]$

Consequently the state space of possible configurations consists of $N \times D \times T$ points where $N, D$, and
$T$ are, respectively, the possible number of nurses, physicians, and technicians. In our small example, the size of the state space is $3 \times 3 \times 3=27$. In general, the state space can be much larger as the number of controllable resources and the number of possible instances of these resources grows.

\section{RESULTS}

This section shows the results of the execution of the first two iterations of the ED controller algorithm discussed in section 2.3 for two experiments that have different interarrival times for the three tracks (see Table 2). The percentage of patients in each track is the same in both experiments but the interarrival times are lower for experiment 2 , which indicates a higher traffic intensity. The warmup time for the experiments was set to 60 minutes and the ED simulator simulates 
24 hours, or a full day, in the modeled ED. Each configuration point was replicated 40 times in the simulator to achieve maximum accuracy and repeatability. Simulation results are reported with $95 \%$ confidence intervals. We assume that $C O S T_{\max }=\$ 6,000.00 / \mathrm{hr}$.

Table 3 shows the results of the controller's first iteration for Experiment 1 (total arrival rate of about 1.4 patients/hr) using the controller algorithm discussed above. Based on the algorithm, the optimal $U(L O S)$ value for this iteration is for configuration $(4,2,5)$. However, due to the $C O S T_{\max }$ constraint of $\$ 6,000 / \mathrm{hr}$, this configuration is not feasible according to Eq. (3). Consequently, based on both the $U(L O S)$ value and the $C O S T_{\max }$ constraint, the optimal ED configuration is actually $(3,3,5)$, which means three nurses, three doctors, and five lab technicians. Using this new configuration as the starting point for the second iteration (Iteration 1) of Experiment 1, we obtain the values in Table 4. The table shows that the optimal configuration for the ED remains at $(3,3,5)$ with a cost of $\$ 5,785.00 / \mathrm{hr}$. The average LOS for this configuration is 54 minutes and the $95 \%$ confidence interval for the LOS is [51 minutes, 57 minutes].

Experiment 2 has an increased total workload intensity of about 1.8 patients/hr. Iteration 0 of the ED Controller for this experiment suggests an optimal configuration of $(4,1,5)$ with a cost of $\$ 5,975.00 / \mathrm{hr}$ (see Table 5). Iteration 1 provides an improved configuration of $(3,1,5)$ with a cost of $\$ 5,521.00 / \mathrm{hr}$ (see Table 6). The average LOS for this configuration is 79 minutes and the $95 \%$ confidence interval for the LOS is [69 minutes, 89 minutes]. Note that the $95 \%$ confidence interval for the $(4,1,5)$ configuration is $[76$ minutes, 96 minutes]. These two configurations are not statistically different in terms of LOS at the $95 \%$ level because the average LOS of one configuration falls in the confidence interval of the other and vice versa (Jain, 1991). However, configuration $(3,1,5)$ is preferable because it uses one less nurse and is less expensive.

Figure 6 shows other details for the optimal configuration for Experiment 2: patient throughput per track (i.e., number of patients in each track processed by the ED during the day), the evolution of throughput over time, the evolution of the number of patients in each track at each hour of the day, and the utilization of various resources (nurse group, physician group, $\mathrm{X}$-ray technicians, and lab technicians).

As shown in Table 7, the ED Controller was able to find an optimal configuration in Experiment 1 that consists of only one nurse less than the maximum number of nurses available and has an average LOS that is lower than the desired LOS of 90 minutes. The cost of this configuration is $4 \%$ lower than the initial configuration that uses the maximum ED resources.

The ED Controller was able to find in the second iteration of Experiment 2 an optimal configuration for the higher intensity workload that is still below the desired LOS goal and at the same time allows a $27 \%$ cost saving with respect to the cost $\$ 7,588.00$ of the initial configuration $(4,3,5)$ for that experiment (see Table 5).

We only showed two iterations of the controller in this paper to illustrate its operation. In practice, the controller will run until the maximum number of iterations is achieved or until it cannot find a better solution.

\section{CONCLUDING REMARKS}

Deciding on the best ED configuration given the ED resource limitations, cost constraints, and unpredictable workload is a complex task. It is extremely difficult for human beings to solve problems of such complexity. Consequently, using the proposed selfmanaged ED to assist charge nurses with the decision making process and managing EDs efficiently may prove to be advantageous.

This paper provides a novel use for a controllerbased autonomic system to implement a self-managed ED that can help improve the ED's performance. This autonomic system is effective in providing best configuration parameters for EDs subject to cost constraints and varying workloads. The experiments reported in this paper clearly show the effectiveness of combining combinatorial search techniques with simulation models when used for QoS control. Not only is the controller able to find the optimal configuration, but it does fast enough to afford its utilization in realworld settings to maintain optimal ED QoS metrics. In fact, simulating 40 replicas of one configuration takes approximately 70 seconds on a $1.73-\mathrm{GHz}$ Intel Core i7 processor.

We are currently working on implementing the results of this paper in a real-world ED and analyzing the controller's effectiveness under cost constraints and actual varying patient arrival rates.

\section{ACKNOWLEDGEMENTS}

The authors would like to thank FlexSim Software Products, Inc. for providing the FlexSim Healthcare Simulation software to complete this research. In particular, the support of Mr. Roger Hullinger and his team at FlexSim Software Products is highly appreciated. 
Table 3: $\vec{C}_{0}$, LOS, utility values, and cost for experiment 1 .

\begin{tabular}{|c|c|c|c|c|c|c|}
\hline \hline$n$ & $d$ & $t$ & LOS (min) & $U($ LOS $)$ & Cost $(\$ / \mathrm{hr})$ & Feasible \\
\hline \hline 4 & 3 & 5 & $56 \pm 3.5$ & 0.963 & $\$ 6,044.00$ & $\times$ \\
\hline 3 & 3 & 5 & $54 \pm 3.0$ & 0.967 & $\$ 5,785.00$ & $\checkmark$ \\
\hline 2 & 3 & 5 & $66 \pm 5.5$ & 0.939 & $\$ 6,155.00$ & $\times$ \\
\hline 4 & 2 & 5 & $53 \pm 2.5$ & 0.968 & $\$ 6,027.00$ & $\times$ \\
\hline 4 & 1 & 5 & $58 \pm 3.5$ & 0.959 & $\$ 4,951.00$ & $\checkmark$ \\
\hline 4 & 3 & 4 & $59 \pm 4.5$ & 0.957 & $\$ 5,993.00$ & $\checkmark$ \\
\hline 4 & 3 & 3 & $57 \pm 3.5$ & 0.961 & $\$ 5,971.00$ & $\checkmark$ \\
\hline \hline
\end{tabular}

Table 4: $\vec{C}_{1}$, LOS, utility values, and cost for experiment 1 .

\begin{tabular}{|c|c|c|c|c|c|c|}
\hline \hline$n$ & $d$ & $t$ & LOS (min) & $U($ LOS $)$ & Cost (\$/hr) & Feasible \\
\hline \hline 3 & 3 & 5 & $54 \pm 3.0$ & 0.868 & $\$ 5,785.00$ & $\checkmark$ \\
\hline 2 & 3 & 5 & $66 \pm 5.5$ & 0.777 & $\$ 6,155.00$ & $\times$ \\
\hline 4 & 3 & 5 & $56 \pm 3.5$ & 0.855 & $\$ 6,044.00$ & $\times$ \\
\hline 3 & 2 & 5 & $58 \pm 3.5$ & 0.841 & $\$ 5,357.00$ & $\checkmark$ \\
\hline 3 & 1 & 5 & $58 \pm 3.5$ & 0.841 & $\$ 6,003.00$ & $\times$ \\
\hline 3 & 3 & 4 & $57 \pm 3.5$ & 0.848 & $\$ 6,053.00$ & $\times$ \\
\hline 3 & 3 & 3 & $55 \pm 3.5$ & 0.861 & $\$ 6,061.00$ & $\times$ \\
\hline \hline
\end{tabular}

Table 5: $\vec{C}_{0}$, LOS, utility values, and cost for experiment 2 .

\begin{tabular}{|c|c|c|c|c|c|c|}
\hline \hline$n$ & $d$ & $t$ & LOS (min) & $U(L O S)$ & Cost $(\$ / \mathrm{hr})$ & Feasible \\
\hline \hline 4 & 3 & 5 & $81 \pm 8.5$ & 0.617 & $\$ 7,558.00$ & $\times$ \\
\hline 3 & 3 & 5 & $80 \pm 7.7$ & 0.629 & $\$ 7,468.00$ & $\times$ \\
\hline 2 & 3 & 5 & $114 \pm 13$ & 0.234 & $\$ 7,642.00$ & $\times$ \\
\hline 4 & 2 & 5 & $92 \pm 14$ & 0.480 & $\$ 7,511.00$ & $\times$ \\
\hline 4 & 1 & 5 & $86 \pm 10$ & 0.556 & $\$ 5,975.00$ & $\checkmark$ \\
\hline 4 & 3 & 4 & $87 \pm 8.5$ & 0.543 & $\$ 7,683.00$ & $\times$ \\
\hline 4 & 3 & 3 & $87 \pm 11$ & 0.543 & $\$ 7,469.00$ & $\times$ \\
\hline \hline
\end{tabular}

Table 6: $\vec{C}_{1}$, LOS, utility values, and cost for experiment 2 .

\begin{tabular}{|c|c|c|c|c|c|c|}
\hline \hline$n$ & $d$ & $t$ & LOS (min) & $U($ LOS $)$ & Cost (\$/hr) & Feasible \\
\hline \hline 4 & 1 & 5 & $86 \pm 10$ & 0.556 & $\$ 5,975.00$ & $\checkmark$ \\
\hline 3 & 1 & 5 & $79 \pm 10$ & 0.641 & $\$ 5,521.00$ & $\checkmark$ \\
\hline 2 & 1 & 5 & $100 \pm 12$ & 0.382 & $\$ 5,205.00$ & $\checkmark$ \\
\hline 4 & 3 & 5 & $81 \pm 8.5$ & 0.617 & $\$ 7,558.00$ & $\times$ \\
\hline 4 & 2 & 5 & $92 \pm 14$ & 0.480 & $\$ 7,511.00$ & $\times$ \\
\hline 4 & 1 & 4 & $83 \pm 9.5$ & 0.593 & $\$ 5,315.00$ & $\checkmark$ \\
\hline 4 & 1 & 3 & $90 \pm 13.5$ & 0.506 & $\$ 5,434.00$ & $\checkmark$ \\
\hline \hline
\end{tabular}

Table 7: Comparison of experiments 1 and 2.

\begin{tabular}{|c|l|c|c|c|}
\hline \hline Experiment & $\begin{array}{l}\text { Optimal Con- } \\
\text { figuration } \\
(n, d, t)\end{array}$ & LOS (in min) & $U($ LOS $)$ & Cost (\$/hr) \\
\hline \hline 1 & $(3,3,5)$ & $54 \pm 3$ & 0.868 & $\$ 5,785.00$ \\
\hline 2 & $(3,1,5)$ & $79 \pm 9$ & 0.641 & $\$ 5,521.00$ \\
\hline \hline
\end{tabular}




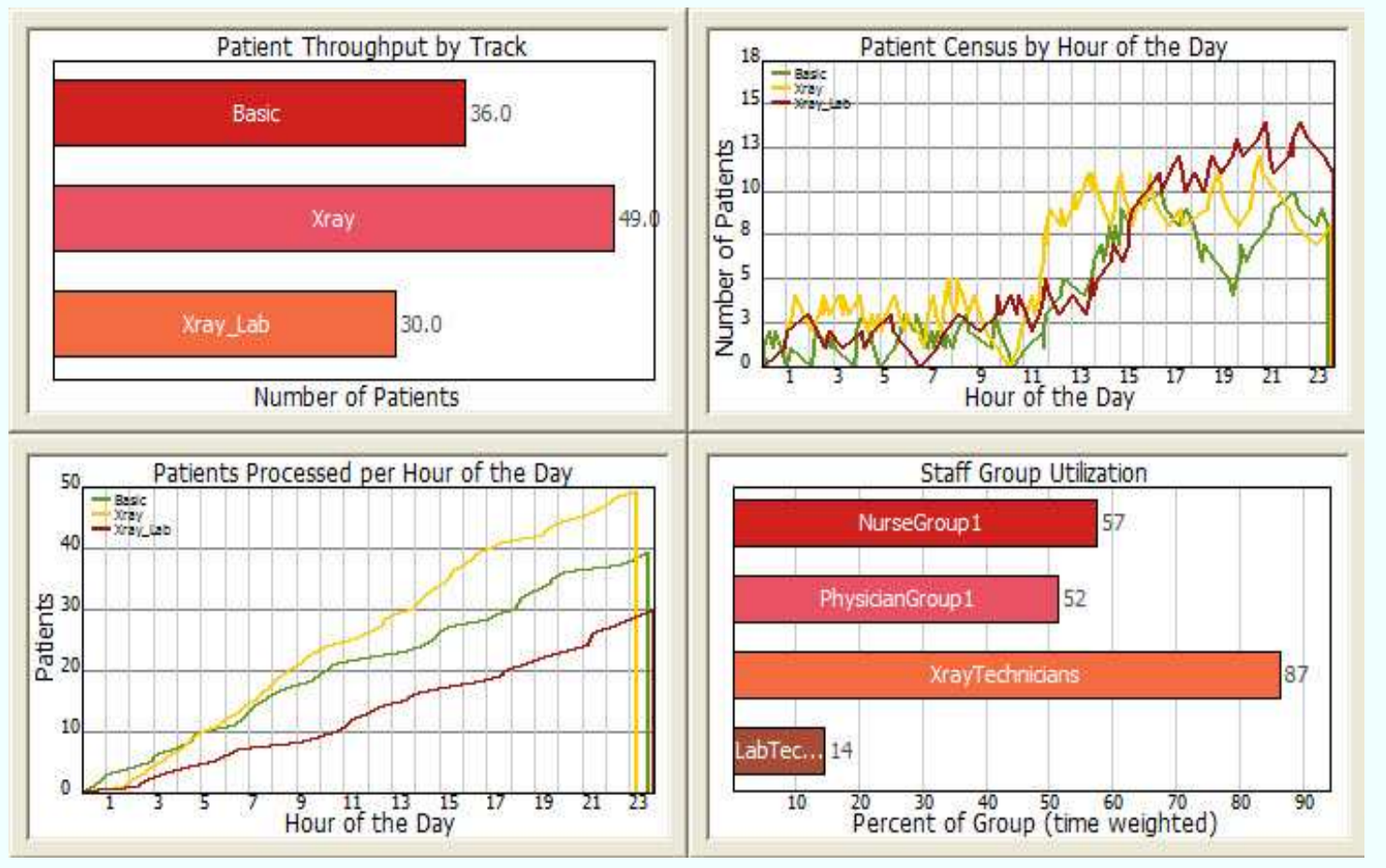

Figure 6: Experiment 2 - ED Optimal Configuration QoS.

\section{REFERENCES}

ACEP (2010). Meeting the challenge of emergency department overcrowding/boarding. American College of Emergency Physicians.

Almomen, S. and Menascé, D. (2011). An autonomic computing framework for self-managed emergency departments. In International Health Informatics Conference, Rome.

Arkun, A., Briggs, W. M., Patel, S., Datillo, P. A., Bove, J., and Birkhahn, R. H. (2009). Emergency department crowding: Factors influencing flow. Western Journal of Emergency Medicine, pages 10-15.

Asplin, B. R., Magid, D. J., Rhodes, K. V., Solberg, L. I., Lurie, N., and Camargo, C. A. (2003). A conceptual model of emergency department crowding. Annuals of Emergency Medicine, pages 173-180.

Bamezai, A., Melnick, G., and Nawathe, A. (2005). The cost of an emergency department visit and its relationship to emergency department volume. American College of Emergency Physicians, pages 483-490.

Fields, W. W. (2003). Calculus, chaos, and other models of emergency department crowding. Annuals of Emergency Medicine, pages 181-184.

Huescher, M. C. and McCann, J. A. (2008). A survey of autonomic computing: Degrees, models, and applications. ACM Computing Surveys, pages 1-28.

Jain, R. (1991). The Art of Computer System Performance Evaluation. John Wiley \& Sons, New York.
Kephart, J. and Chess, D. (2003). The vision of autonomic computing. IEEE Internet Computing, pages 41-50.

Parasuraman, R., Sheridan, T. B., and Wickens, C. D. (2000). A model for types and levels of human interaction with automation. IEEE Systems, Man, and Cybernetics Society, 30:286-297.

Rayward-Smith, V., Osman, I., and Reeves, C. (1996). Modern Heuristic Search Methods. John Wiley \& Sons.

Roberts, L., Johnson, D. C., Shanmugam, D. R., Malhotra, S., Zinkgraf, D. S., Young, M., Putnam, J., and Cameron, L. (2006). Computer simulation and sixsigma tools applied to process improvement in an emergency department. Institue of Industrial Engineers Society for Health Systems.

Welch, S., Augustine, J., Camargo, C. A., and Reese, C. (2006). Emergency department performance measures and benchmarking summit. Academic Emergency Medicine, pages 1074-1080. 